\title{
Videojuegos, práctica de actividad física, obesidad y hábitos sedentarios en escolares de entre 10 y 12 años de la provincia de Granada \\ Videogames, physical activity practice, obesity, and sedentary habits in schoolchildren aged 10 to 12 years old in the province of Granada \\ Antonio Miguel Lozano-Sánchez, Félix Zurita-Ortega, José Luis Ubago-Jiménez, Pilar Puertas-Molero, Irwin Ramírez-Granizo, Jose Iván Núñez-Quiroga \\ Universidad de Granda (España)
}

Resumen: La actividad física constituye uno de los pilares fundamentales para el correcto desarrollo tanto físico como psicológico del ser humano, pero especialmente en los más jóvenes, ya que es considerada un medio esencial para la prevención de patologías y mejora de la salud. Esta investigación de tipo descriptivo y corte transversal se ha realizado en una muestra de 261 niños y niñas de entre 10 y 12 años, de colegios de la provincia de Granada y alrededores. Los objetivos planteados resultan de gran trascendencia en edad escolar ya que fundamentan la base principal del desarrollo de los jóvenes a edades tempranas en términos de salud y hábitos de ocio digital asociados al sedentarismo. Los resultados han determinado que los casos de sobrepeso en escolares eran escasos y que un importante número de jóvenes realiza práctica de ejercicio físico fuera del horario escolar. En cuanto a uso de videojuegos, casi la totalidad de los jóvenes tiene un hábito de consumo de ellos aunque se han obtenido índices mínimos en cuanto a problemática severa de adicción digital. Tras la asociación de estas variables se ha podido ver cómo estos factores normalmente generalizados como principales factores incidentes en la obesidad infantil, se están considerando como elementos a cambiar debido a los cambios producidos en los tiempos de ocio a favor de la actividad física y a las innovaciones llevadas a cabo en plataformas digitales activas orientadas a la combinación del fenómeno «gamer» y la movilidad.

Palabras clave: Actividad física, Educación Primaria, videojuegos, obesidad, salud, sedentarismo.

Abstract: Physical activity constitutes one of the fundamental pillars for the correct physical and psychological development of human beings, especially in young people, since it represents an essential means for the prevention of pathologies and improvement of health. This cross-sectional and descriptive research was carried out in a sample of 261 children aged between 10 and 12 years old enrolled in schools in the province of Granada and the surrounding areas. The objectives presented are greatly significant in school age because they are the basis for the development of young people at an early age, with a focus on health and digital leisure habits associated with sedentary lifestyle. The results determined that cases of overweight in schoolchildren were scarce and that a significant number of young people practice physical exercise out of the school hours. As for video games, almost all children play them, although they have obtained minimum rates in terms of severe digital addiction problems. After assessing the association of these variables, it was possible to verify how these factors, generally recognized to have a huge impact on childhood obesity, are considered as the elements needing to be modified due to shift in leisure time in favor of physical activity and the innovations brought on active digital platforms oriented towards the combination of the «gamer» phenomenon and mobility.

Key words: Physical activity, Primary Education, video-games, obesity, health, sedentary lifestyle.

\section{Introducción}

En las últimas décadas, los altos índices de obesidad se han consolidado como uno de los principales problemas de la sociedad a nivel mundial (Gao, Lee, Pope y Zhang, 2016; Martínez et al., 2017). Además, estos casos multitudinarios de obesidad se encuentran estrechamente vinculados a hábitos de sedentarismo, considerado igualmente otro problema de relevante gravedad, ya que cada vez es más acentuada la tendencia a llevar a cabo actividades de tipo sedentario en tiempo de ocio. Muchos expertos en la problemática confluyen sus investigaciones en el fuerte auge del desarrollo tecnológico que viene produciéndose desde hace dos décadas, dónde en los hogares es cada vez más frecuente encontrar la presencia de dispositivos electrónicos tales como smartphones, tabletas digitales, ordenadores y videoconsolas, lo cual parece explicar que el sedentarismo viene ahora apellidado como «sedentarismo tecnológico» y que éste conlleva a la tan afamada obesidad (Castro et al., 2015).

Ante esta situación, resulta imprescindible analizar la bibliografía reciente respecto al sobrepeso y la obesidad, así como su relación con el uso intensivo de videojuegos. En primer lugar, la Organización Mundial dela Salud (2012) define estos términos como una acumulación anormal o excesiva de grasa que puede ser perjudicial para la salud. En cuanto a los determinantes fundamentales de los factores de riesgo que dan lugar a casos de obesidad, autores como Martínez, Villarino, García, Calle y Marrodán (2013) recogen como principales causantes las familias dado que la obesidad puede llevar consigo un componente genético y hereditario-; los cambios producidos en la dieta -conducentes a una mayor ingesta dónde hay mayor presencia de grasas, azúcares añadidos

Fecha recepción: 20-11-17. Fecha de aceptación: 14-05-18 Antonio Miguel Lozano-Sánchez amlozano95@correo.ugr.es y grasas saturadas junto con una reducción del consumo de carbohidratos complejos y fibra junto con la de frutas y verduras-; y sobretodo en niños y adolescentes, la inactividad física, la cual juega un papel predominante.

La actividad física se ha convertido en un componente de especial relevancia para hacer frente a esta problemática. Existen evidencias científicas que indican que llevar una vida activa reduce el sobrepeso y, consecuentemente la obesidad, ya que el ejercicio mantiene una relación directa con el peso y la composición corporal (Trejo, Jasso, Mollinedo y Lugo, 2012). En la población escolar, existe una dura confrontación entre la práctica de movilidad física activa y el tiempo dedicado al ocio digital, ya que en numerosos casos existe una tendencia desmesurada de uso de videojuegos y otros hábitos de consumo de pantalla, lo cual aleja a satisfacer los treinta minutos diarios de ejercicio físico necesarios para llevar una vida saludable en términos de movilidad activa propuesto por la OMS (Poletti y Barrios, 2007). De ello deriva la alarmante cifra de casos de obesidad infantil diagnosticada entre la población infantil y juvenil en España, rondando el 40\% en estudios como el propuesto por Sánchez-Cruz, Jiménez-Moleón, Fernández-Fernández y Sánchez (2013).

Sabiendo el vínculo existente entre ejercicio físico y salud, se plantea la reflexión sobre si el Sistema Educativo puede tomar cartas en el asunto para tratar de reducir todo lo posible los casos de obesidad desde edades tempranas. Son muchas las investigaciones que identifican la escuela como un lugar adecuado para promover la actividad física (Tornero, Sierra, Carmona, y Gago, 2015). Ésta se sitúa como pilar fundamental para mantener una salud adecuada, derivando con ello las buenas actitudes hacia las personas obesas. Es por tanto el área de Educación Física y su currículum la que debe centrarse en no sólo llevar a cabo la práctica de actividad física, sino la de crear y concienciar sobre los hábitos de vida saludables.

No son pocas las fuentes que destacan el gran avance que la tecno- 
logía ha sufrido en los últimos años de una manera abrumadora (Duque y Vásquez, 2013). Gracias a este progreso, se han conseguido extraordinarios avances en el ámbito científico, pero lo más importante, es que ha conseguido llegar hasta la gran mayoría de nuestros hogares. El auge de las Tecnologías de la Información y Comunicación (TIC) ha hecho que los niños y adolescentes se perfilen como sujetos potencialmente familiarizados y atraídos por el uso de las pantallas. Esta atracción genera en los docentes la necesidad de hacer uso de las mismas para educarlos y formarlos. Acercándonos a este nuevo horizonte educativo, cabe señalar los conocidos «Nacidos Digitales» o «Generación Interactiva» como una generación que ha nacido y crecido frente a las pantallas (Bringué y Sádaba, 2009). Como recurso a esta necesidad metodológica de incorporar las nuevas tecnologías a las prácticas educativas, surgen los «Exergames», definidos por Staiano y Calvert (2011) como juegos digitales que combinan el ejercicio físico y el juego, mejorando el estado de salud de los que lo practican además de proporcionarles beneficios sociales y académicos. Gracias a ellos podremos mejorar sustancialmente las clases de Educación Física y conseguir que los alumnos continúen con la práctica activa en casa de una manera divertida.

Este nuevo tipo de plataformas que proporcionan al mismo tiempo ejercicio y juego han emergido como una herramienta innovadora para combatir la crisis de los altos índices de obesidad que sufre la sociedad actual (McGinnis, Gootman y Kraak, 2006). Autores como Beltrán, Valencia y Molina (2011) confirman que en estos últimos años se ha dado un paso más en el ámbito de los videojuegos, dejando atrás la concepción del videojuego pasivo que daba lugar al sedentarismo, para dar lugar a la aparición de una nueva generación de videojuegos que implican actividad física y movimiento. Para ello, se establece una relación entre el movimiento de los jugadores con la realidad virtual que aparece en la pantalla a través de distintos utensilios, a destacar entre ellos controladores con sensores ópticos, que permite apuntar a objetos en la pantalla, y un acelerómetro, que se encarga de detectar los movimientos del jugador en tres dimensiones y trasladarlos del mismo modo a la pantalla (Beltrán et al., 2011).

Tras este breve acercamiento a los videojuegos activos, resulta relevante conocer qué plataformas han desarrollado su funcionamiento. Para ello, Chacón (2014) establece una relación de las distintas que hoy en día nos encontrar en el mercado y que han apostado por este tipo de videojuegos como pioneros en la categoría «Exergames»:

Tabla 1.

Plataformas desarrolladoras de videojuegos activo.

Nintendo Videoconsola producida por Nintendo en 2006. Su característica más destacable es Wii el "WiiMote", un controlador inalámbrico que se usa como puntero hacia la

Wi pantalla además de detectar los movimientos del jugador.

Firmada por la marca Sony y lanzada al mercado en 2010. Utiliza un mando

inalámbrico con sensor de movimiento y una cámara conocida como "PlayStation

Move Eye" que se encarga de detectar la posición de dicho controlador. Controlador creado y desarrollado por Microsoft para Xbox lanzada a meado Controlador creado y desarrollado por Microsolt para Xbox lanzada al mercado en Kinect mandos o controladores, se maneja mediante una interfaz de reconocimiento de gestos y comandos de voz.

En cuanto las ventajas e inconvenientes que este tipo de plataformas conllevan tanto en el ámbito escolar como fuera de él, González, Navarro, Castillo, Quirce y Vera (2012), entre otros, señalan que la puesta en marcha deactividades en las que se haga uso de los «Exergames» posibilitará el trabajo de contenidos del currículum como son las habilidades motrices o la lateralidad. Además, al mismo tiempo que se trabaja estos contenidos, se desarrolla la competencia digital. Sin embargo, no hay que olvidar que este tipo de videojuegos activos están pensados para la puesta en práctica de manera individualizada en su mayoría o hasta cuatro jugadores en algunos de los casos, por lo que tienden a desempeñarse dentro de un aprendizaje excesivamente individualizado (Baracho, Gripp y De Lima, 2011).

Respecto a su uso fuera del aula, se trabajarían los mismos componentes que se han señalado anteriormente dentro del aula, pero se le añadiría una de las premisas fundamentales que se pretende fomentar con la puesta en marcha de esta investigación: la creación de hábitos saludables. El empleo de estos videojuegos en casa hará que se produzca un giro radical desde un jugador pasivo hacia un jugador activo, mejorando por tanto los niveles cardiovasculares y músculo - esqueléticos, así como la disminución de la presencia de casos de obesidad o sedentarismo (Chacón, Castro, Zurita, Espejo y Martínez, 2016).

Con el propósito de crear una base de sustentación en la que se apoye todo lo anteriormente descrito y servir como guía para la posterior investigación, se establecen los siguientes objetivos:

- Analizar el grado de obesidad en los escolares, la frecuencia de práctica de actividad física en horario extraescolar, así como la dedicación al disfrute del ocio digital.

- Relacionar los hábitos sedentarios a edades tempranas con la dedicación a videojuegos al igual que con los perfiles de obesidad.

\section{Método}

\section{Diseño}

Se realizó un estudio no experimental, de tipo descriptivo y corte trasversal. Asimismo, el diseño fue analítico y relacional, al concretar relaciones entre todas las variables.

\section{Participantes}

La muestra estuvo constituida por 261 niños y niñas de entre $10 \mathrm{y}$ 12 años, representando un 55,6\% (n=145) el género masculino y un $44,4 \%$ ( $n=116$ ) al femenino, de los dos últimos cursos de la etapa de Educación Primaria, pertenecientes tanto a colegios públicos como privados de la provincia de Granada así como de diversos municipios pertenecientes a la misma.

\section{Instrumentos yvariables}

Las variables empleadas para llevar a cabo esta investigación fueron las siguientes:

- Edad.

- Género, bien sea masculino o femenino. IMC.

Peso y altura, para estipular el grado de obesidad en función del

- IMC categorizado (Obesidad), se valoró el Índice de Masa Corporal, categorizado en cuatro niveles (1=Bajo Peso, 2=Normopeso, $3=$ Sobrepeso y 4=Obeso), utilizando el estudio enKid (Serra et al., 2003).

- Práctica de actividad física o deporte, considerando si se realizaba más de 3 horas de práctica físico-deportiva extraescolar semanalmente, categorizado en sí o no.

- Frecuencia de uso de videojuegos, con el fin de determinar las horas diarias y semanales que invertían los escolares en ocio digital de pantalla sedentario.

Para la recogida de datos que posteriormente se ha empleado para la elaboración de esta investigación, se utilizaron los siguientes instrumentos:

Cuestionario de Experiencias Relacionadas con los Videojuegos (CERV), el cual fue validado por Chamarro et al. (2014) en adolescentes. El test se compone de 17 ítems de connotación negativa, los cuales son puntuados a través de una escala Likert de cuatro opciones ( $1=$ Casi Nunca; 2 =Algunas veces; 3 = Bastantes veces; 4 = Casi siempre). Este instrumento permite valorar el uso problemático de videojuegos a través de una sumatoria que categoriza la variable en terciles de la siguiente forma: Sin problemas: de 17 a 25; Problemas potenciales: de 26 a 38; Problemas severos: de 39 a 68, quedando así representado el nivel de uso problemático de videojuegos.

- Báscula, con el propósito de pesar a los alumnos y conocer su peso.

- Cinta métrica, para medir a los alumnos y conocer su estatura.

- IMC, con el fin de determinar los índices de obesidad bien sean bajo peso, normopeso, sobrepeso u obesidad, según el estudio enKid (Serra et al., 2003).

\section{Procedimiento}

En primer lugar, se informó a los centros dónde se proyectó llevar 
a cabo la realización de los cuestionarios oportunos sobre el propósito de los mismos solicitando acto seguido su aprobación asegurando la total confidencialidad y el anonimato de los datos obtenidos. Como justificante para su aprobación, se aportó una carta informativa remitida por la Facultad de Ciencias de la Educación de la Universidad de Granada desde el departamento de Didáctica de la Expresión Musical, Plástica y Corporal. Tras el consentimiento por parte de todos los centros, se procedió a la elaboración de los cuestionarios, incluyendo las versiones validadas anteriormente señaladas. A la hora de proceder a la elaboración de los cuestionarios, se informó previamente tanto al director como al tutor/docente de los grupos que iban a realizarlo ya que este debía ser realizado en horario escolar. Previamente al reparto de los documentos, se informó a todo el alumnado sobre el motivo de la elaboración de dicho material y las instrucciones para ser rellenado correctamente, dejando claro desdeel primermomento que se garantizaba la total confidencialidad de los datos que ellos iban a reflejar y solicitando además el consentimiento de los alumnos a la realización de dicho documento. Durante el proceso de elaboración por parte de los alumnos, se resolvieron todo tipo de cuestiones que pudieron surgir y se explicaron algunos conceptos que resultaron confusos. Asimismo, se ha informado a los centros educativos de los resultados obtenidos en la investigación.

\section{Análisis de los datos}

El análisis de los datos se realizó empleando el software estadístico IBM SPSS 22.0. Se emplearon frecuencias y medias para los análisis estadísticos descriptivos básicos y correlaciones bivariadas para determinar las relaciones entre las variables. Se empleó la prueba de Chicuadrado de Pearson ( $p>.05)$ para determinar la significatividad de las relaciones.

\section{Resultados}

Los descriptivos básicos de la muestra estudiada quedó representada por un $55,6 \%$ de alumnos masculinos $(n=145$ ) y un $44,4 \%$ femeninos (n=116). Del mismo modo la distribución de los sujetos según la edad quedó reflejada por un $34,4 \%$ de alumnos/as de 10 años ( $\mathrm{n}=90$ ), un 47,5\% de 11 años ( $n=124)$ y un 18,0\% de 12 años ( $n=47)$.

Según los resultados obtenidos tras el estudio de los niveles de índice de masa corporal entre los participantes (Tabla 2) se obtuvo que un 55,6\% de los estudiantes de los últimos cursos de la etapa de Educación Primaria presentan bajo peso, mientras que un 38,7\% presentan unos índices de peso normal. Los casos de sobrepeso se vieron representados por un 5,4\% al mismo tiempo que el de obesos se dio con un

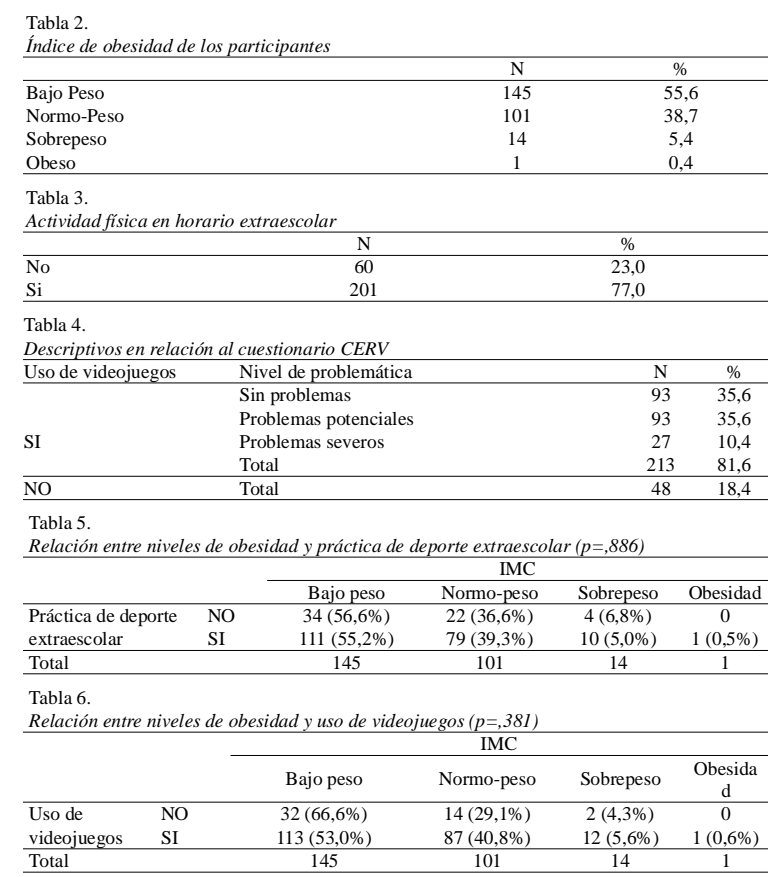

$0,4 \%$ con la presencia de un solo caso.

El porcentaje de participantes que no realizaba práctica de ejercicio físico fuera del horario escolar (Tabla 3) quedó representado por un 23,0\% frente a un 77,0\% que sí reconocía realizarlo de manera periódica. Del mismo modo, tras el análisis del uso y hábito de consumo de videojuegos (Tabla 4) se obtuvo que un $18,4 \%$ de los participantes no tenía hábito de ocio digital, mientras que un 81,6\% sí reconocía jugar frecuentemente a videojuegos. Los niveles de uso problemático de consumo de videojuegos denotaron que un $35,6 \%$ de los participantes no presentaban problemas derivados de ello, un 35,6\% presentaba problemas potenciales y un $10,4 \%$ mostraba problemas severos derivados de un uso excesivo de videojuegos.

Tras establecer relaciones entre los niveles de obesidad de los participantes con la práctica de ejercicio físico en horario extraescolar(Tabla 5) se han obtenido escasas diferencias estadísticamente significativas entre ellos ( $p=, 886)$, mostrando valores similares tanto para los activos en la práctica física como los indiferentes. De igual modo, la relación entre los niveles de obesidad y el uso de videojuegos (Tabla 6) ha demostrado que se presentaban ligeras diferencias entre ellos ( $p=, 381$ ), aunque sí se puede apreciar una mayor estabilidad en cuanto al balance de casos de bajo peso y normo-peso.

\section{Discusión}

Resulta de especial relevancia el estudio de los objetivos planteados en esta investigación en edad escolar ya que fundamentan la base de sustentación principal del desarrollo de los jóvenes a edades tempranas en términos de salud y hábitos de ocio digital asociados al sedentarismo. De este modo, han sido numerosas las investigaciones que han enfocado su visión hacia el estudio de estos parámetros destacando los llevados a cabo por Aguilar et al. (2011); Beltrán et al. (2011); Best (2013) o Chacón et al. (2015), entre otros.

En primer lugar, el estudio de los niveles deíndice de masa corporal (IMC) mostró que más de la mitad de los participantes mostraban cotas de bajo peso. No obstante, estudios como el elaborado por Pérez, Sandoval, Schneider y Azula (2008) o Serra et al. (2003), encuentran este parámetro como el minoritario entre los posibles, siendo el más común el normopeso. Esta diferencia se debe a que estos estudios dedican su análisis a toda la etapa de la educación primaria de manera globalizada, mientras que en la presente se toman como referencia los dos últimos cursos donde los alumnos están sufriendo un proceso de crecimiento importante y los cálculos oportunos tienden a dar resultados a la baja.

Por otro lado, 7 de cada 10 escolares afirmaban realizar práctica de ejercicio físico fuera del horario escolar. González y Portolés (2014) obtuvieron resultados que corroboran estos datos, elaborando un estudio en el que se reflejó que la mayoría de la muestra analizada realizaba actividad física y deportiva extraescolar de manera periódica añadiendo que deportes como el fútbol o el baloncesto son los más demandados. Este alto índice de práctica deportiva en tiempo de ocio se debe a que los escolares a estas edades todavía sienten inquietud por el deporte y la actividad física y no han sufrido aun el cambio madurativo propio hacia la adolescencia la cual provoca un considerable descenso de la práctica deportiva, siendo considerablemente más pronunciado en el género femenino. Según la última Encuesta Nacional de Salud (Ministerio de Sanidad, Servicios Sociales e Igualdad, 2014), es a partir de los 9 años cuando este hábito de práctica de actividad física extracurricular comienza a tener especial relevancia. Dicho informe también justifica por tanto los datos obtenidos en este estudio acerca de la relación de la edad de los participantes de la muestra con la práctica de ejercicio físico en horario extraescolar, ya que todos los escolares comprendían edades entre los 10 y los 12 años, rango de edad en la que la práctica física es homogénea.

En cuanto al uso y hábito de consumo de videojuegos, nos encontramos frente a una amplia mayoría de jóvenes que reconocían jugar frecuentemente a videojuegos en tiempo de ocio. En la investigación llevada a cabo por Ferrer y Ruiz (2005) en la que se pretendía conocer los hábitos básicos de los niños en la etapa de Educación Primaria en 
relación con los videojuegos, se obtuvo un resultado similar donde 9 de cada 10 escolares reconocían dedicar parte de su tiempo de ocio al uso de videojuegos. Estos datos resultan de gran significatividad ya que confirman el alto grado de motivación que suponen las nuevas tecnologías para los escolares y fortalecen la amplitud del fenómeno «gamer» así como la necesidad de desarrollar mecanismos educativos en los que la influencia del ocio electrónico afecte de manera positiva al desarrollo educativo de los más jóvenes tal y como lo fundamentan investigaciones como la realizada por Beltrán et al. (2011).

Como consecuencia a este elevado número de participantes asiduos a los videojuegos, frente a todo pronóstico, los resultados obtenidos en cuanto a problemática asociada a videojuegos denotaron que 1 de cada 10 jugadores padecían problemas severos de consumo digital, dato que contrasta con los resultados obtenidos por Fogel, Miltenberger, Graves y Koehler (2010) dónde se triplicaba la proporción de casos de problemática severa con respecto al uso de videojuegos. Dicha variación puede ser dada a que a la muestra analizada por estos autores se le añadían participantes de edades correspondientes a los primeros cursos de la Educación Secundaria, etapa en la que el número de horas dedicadas al ocio digital incrementa dado el mayor catálogo de videojuegos ofertado para este rango de edades.

Retomando lo recogido acerca de los niveles de índice de masa corporal, en su asociación con la práctica de actividad física en horario extraescolar, se obtuvieron variaciones escasas entre los que practican actividad física de manera regular y los que lo niegan. Estos resultados, pese a contrastar con estudios como los llevados a cabo por RamiroGonzález, Sanz-Barbero y Royo-Bordonada (2017) o Díaz (2017), en los que sitúan la obesidad infantil como una problemática a nivel nacional de forma generalizada, se deben a que en estos colegios se ha apostado de manera eficaz por la importancia de llevar a cabo una dieta equilibrada basada en el modelo de dieta mediterránea, elaborando planes internos para controlar tanto el hábito alimenticio en el horario escolar como fuera de él, motivando al alumnado para su consecución y logro y concienciando a toda la comunidad educativa sobre la problemática actual de obesidad infantil asociada a la ingesta de alimentos no saludables y el sedentarismo, objetos de estudio para esta investigación. Con ello, se ha conseguido compensar la carencia de hábito de práctica de ejercicio físico regular en tiempo extraescolar con la modificación de la dieta orientada a una más saludable.

Esta justificación se puede aplicar del mismo modo a las escasas diferencias obtenidas entre la relación de los niveles de obesidad con el consumo de ocio digital, añadiéndole a ésta, el gran auge que actualmente se viene produciendo del fenómeno «Exergame» o videojuegos activos, en los que se producen beneficios saludables de manera inconsciente en aquellos jugadores que los ponen en práctica ya que se combinan la movilidad física junto con el disfrute digital. Sun (2013) recoge en su investigación cómo esta nueva tecnología activa genera motivación especialmente en los alumnos de Educación Primaria y han resultado ser un punto de inflexión para aquellos que gastaban su tiempo de ocio en rutinas sedentarias, siendo los «Exergames» un medio notablemente válido para promover la actividad física.

En relación a lo expuesto, este estudio alcanza como principales conclusiones:

Actualmente se están llevando a cabo medidas para tratar de resolver la problemática de obesidad en escolares, la cual es un hecho verídico, pero analizando muestras concretas podemos ver cómo las soluciones surgen efecto. En primer lugar, tras el análisis de los niveles deíndice de masa corporal en escolares de entre 10 y 12 años se obtuvo que más de la mitad de los participantes mostraban cotas de bajo peso. Este dato, pese a contrastar con los estudios señalados en el marco teórico, recoge las primeras observaciones de que los casos de obesidad se van reduciendo, pese a no ser el rango de peso ideal debido a su etapa de crecimiento y desarrollo corporal. Como agregado a la solución del problema, se recogió que la mayoría de escolares a estas edades realiza ejercicio físico fuera del horario escolar de manera periódica.

En cuanto al análisis de los factores determinantes que van ligados a la obesidad, la asociación de ésta en función del índice de masa corporal de los participantes con la regularidad de práctica de actividad física y el consumo de ocio digital ha demostrado ligeras diferencias entre sí, justificando con ello la no existencia generalizada de estos dos últimos factores como primeros causantes de la obesidad infantil. Tras el estudio de los casos, se ha podido ver cómo la actividad física juega un papel relevante entre los rangos de edad que nos encontramos, 10 y 12 años, y que se posiciona como una preferencia a la hora de establecer los tiempos de ocio entre los escolares. Del mismo modo, el marco teórico empleado ha justificado el gran beneficio de las mejoras producidas en el ámbito de los videojuegos gracias a los «Exergames», ya que pese a haber obtenido una clara mayoría de casos que reconocen jugar a ellos, estas plataformas se posicionan como un elemento primordial a la hora de combinar el ocio digital con la movilidad, generando un avance relevante en el ámbito de la actividad física ya que su implementación en las aulas generaría un claro punto de inflexión en la materia impulsado por la motivación generada en el alumnado.

\section{Limitaciones y prospectiva de estudios futuros}

Con la elaboración de esta investigación, las principales limitaciones han surgido a la hora de proceder a la realización de los cuestionarios dado el escaso tiempo proporcionado por los colegios para la realización de los mismos. De igual modo, el diseño llevado a cabo de tipo descriptivo y corte transversal tan sólo ha permitido observar asociaciones entre las variables pero no elaborar relaciones causa-efecto, algo factible con un diseño longitudinal.

Como perspectivas futuras, este estudio pretende ser un punto de partida para una investigación posterior en la que se pretende desarrollar una propuesta de implementación en el aula en la que los escolares encuentren un equilibrio atractivo entre el uso y hábito de consumo de ocio digital y la práctica regular de ejercicio físico en horario no lectivo, llevando consigo una estabilización de los índices de masa corporal adecuados para la edad en la que se encuentran. Esta proposición resulta interesante llevarla a cabo desde los «Exergames», ya que se sitúan como una plataforma digital novedosa que resulta de gran atractivo para los jóvenes e invita a la práctica física mediante el juego. En definitiva, se trata de aprovechar el gran auge de las nuevas tecnologías para promover el movimiento de los más pequeños combinándolo con la diversión y entretenimiento, luchando de manera efectiva contra los hábitos sedentarios y la obesidad.

\section{Agradecimientos}

Este trabajo de investigación ha sido llevado a cabo durante el trascurso de una beca de iniciación a la investigación para estudiantes de grado perteneciente al Plan Propio de Investigación de la Universidad de Granada en el año 2017. Agradecer a dicha institución por hacer posible la realización de este estudio, así como su apoyo y colaboración.

\section{Referencias}

Aguilar, M., González, E., García, C., García, P., Álvarez, J., Padilla, C., González, J. y Ocete, E. (2011). Obesidad de una población de escolares de Granada: evaluación de la eficacia de una intervención educativa. Nutrición Hospitalaria, 26(3), 636-641.

Baracho, A, Gripp, J., y de Lima, R. (2011). Exergames: la Escuela, Educación Física y cultura en digital. Brazilian Journal of Sports Sciences, 34 (1), 1-14

Beltrán, V., Valencia, A. y Molina, J. (2011). Los videojuegos activos y la salud de los jóvenes: revisión de la investigación. Revista Internacional de Medicina y Ciencias de la Actividad Física y el Deporte, 10 (41), 203-219.

Best, J. (2013). Exergaming in youth: Effects on physical and cognitive health. Zeitschriftür Psychologie, 221(2), 72-78.

Bringué, X., y Sádaba, C. (2009). Nacidos digitales: una generación frente a las pantallas. Madrid: Ediciones Rialp.

Castro, M., Martínez, A., Zurita, F., Chacón, R., Espejo, T. y Cabrera, 
A. (2015). Uso de videojuegos y su relación con las conductas sedentarias en una población escolar y universitaria. Journal for Educators, Teachers and Trainers, 6(1), 56-66.

Chacón, R. (2014). Los «Exergames» como posibilidad emergente y alternativa, en el ámbito de la actividad física y la salud (Trabajo fin de Grado). Facultad de Ciencias de la Educación, Granada, España.

Chacón, R., Castro, M., Zurita, F., Espejo, T. y Martínez, A. (2016) Videojuegos activos como recurso TIC en el aula de Educación Física: estudio a partir de parámetros de ocio digital.Digital Education Review, 29, 112-123.

Chacón, R., Espejo, T., Cabrera, A., Castro, M., López, J., Zurita, F. (2015). «Exergames» para la mejora de la salud en niños y niñas en edad escolar: estudio a partir de hábitos sedentarios e índices de obesidad. Revista Latinoamericana De Tecnología Educativa, 14(2), 39-50.

Chamarro, A., Carbonell, X., Manresa, J., Muñoz, R., Ortega, R., López, M., Batalla, C. y Torán, P. (2014). El Cuestionario de Experiencias Relacionadas con los Videojuegos (CERV). Un instrumento para detectar el uso problemático de videojuegos en adolescentes españoles. Adicciones: Revista de socidrogalcohol. 26(4), 303-311.

Díaz, J. (2017). Childhood obesity: ¿Prevention or treatment? Anales de Pediatría, 86(4), 173-175.

Duque, E. y Vásquez,A. (2013). NUI para la educación. Eliminando la discriminación tecnológica en la búsqueda de la Inclusión Digital. Centro de Investigaciones, Corporación Universitaria Americana.

Ferrer, M. y Ruiz, J. (2006). Uso de videojuegos en niños de 7 a 10 años. Una aproximación mediante encuesta. ICONO 14, 4(1), 115.

Fogel, V., Miltenberguer, R., Graves, R. y Koehler, S. (2010). The effects of exergaming on physical activity among inactive children in a physical education classroom. Journal of applied behavior analysis, 43(4), 591-600.

Gao, Z., Lee, J., Pope, Z. y Zhang, D. (2016). Effect of Active Videogames on UndeservedChildren's Classroom Behaviors, Effort, and Fitness. Games for Health Journal, 5(5), 318-324.

González, C., Navarro, V., Castillo, J. M., Quirce, C., y Vera, S. (2012). Videojuegos yoportunidades para el aprendizaje: Videojuegos activos y promoción de hábitos saludables para menores en situación de enfermedad. Las Palmas de Gran Canaria: EDUTEC 2012

González, J. y Portolés, A. (2014). Actividad física extraescolar: relaciones con la motivación educativa, rendimiento académico y conductas asociadas a la salud. Revista Iberoamericana de Psicología del Ejercicio y el Deporte, 9(1), 51-65.

Martínez, J., Villarino,A., García, R., Calle, M., y Marrodán, M. (2013). Obesidad infantil en España: hasta qué punto es un problema de salud pública o sobre la fiabilidad de las encuestas. Nutrición Clínica y Dieta Hospitalaria, 33, 80-88.
Martínez, M., Rico, S., Rodríguez, F., Gil, G., Santano, E., y Calderón, J. (2017). Influencia de los hábitos de ocio sedentario en el desarrollo de sobrepeso y obesidad en una población de escolares. NURE Investigación, 14(87), 1-8

McGinnis, J., Gootman, J. y Kraak, V.(Eds.) and the Committee on Food Marketing and the Diets of Children and Youth, Food and Nutrition Board, Board on Children, Youth, and Families, Institute of Medicine of the National Academies (2006). Food marketing to children and youth: Threat or opportunity? The National Academies Press. Washington, DC.

Ministerio de Sanidad, Servicios Sociales e Igualdad (2014). Encuesta Nacional de Salud. España 2011/12. Actividad física, descanso y ocio. Serie informes monográficos, 4.

Organización Mundial de la Salud (Mayo de 2012). Obesidad y sobrepeso. Nota descriptiva No 311. Recuperado el día 05 de marzo del 2017, desde: http://www.who.int/mediacentre/factsheets/ fs311/es/

Pérez, E., Sandoval, M., Schneider, S. y Azula, L. (2008). Epistemología del sobrepeso y la obesidad en niños y adolescentes. Revista de Posgrado de la VIa Cátedra de Medicina, 179, 16-20.

Poletti, H. y Barrios, L. (2007). Sobrepeso, obesidad, hábitos alimentarios, actividad física y uso de tiempo libre en escolares de Corrientes (Argentina). Revista cubana de pediatría, 79(1)

Ramiro-González, M., Sanz-Barbero, B. y Royo-Bordonada, M. (2017). Childhood excess weight in Spain from 2006 to 2012. Determinants and parental misperception. Revista Española de Cardiología, 70(8), 656-663.

Sánchez-Cruz, J. Jiménez-Moleón, J., Fernández-Quesada, F. y Sánchez, M. (2013). Prevalencia de obesidad infantil y juvenil en España en 2012. Revista española de cardiología, 66(5), 371-376.

Serra, L., Ribas, L., Aranceta, J., Pérez, C., Saavedra, P. y Peña, L. (2003). Obesidad infantil y juvenil en España. Resultados del Estudio enKid(1998-2000). Medicina Clínica Barcelona, 121(19), 725732.

Staiano, A. y Calvert, S. (2011). Exergames for Physical Education Courses: Physical, Social and Cognitive Benefits. Child Development Perspectives, 5 (2), 93-98.

Sun, H. (2013). Impact of Exergames on physical activity and motivation in elementary school students: A follow-up study. Journal of Sport and Health Science, 2(3), 138-145.

Tornero, I., Sierra, A., Carmona, J. y Gago, J. (2015). Implicaciones didácticas para la mejora de la imagen corporal y las actitudes hacia la obesidad desde la Educación Física. Retos: Nuevas tendencias en Educación Física, Deportes y Recreación, 27, 146-151.

Trejo, P., Jasso, S., Mollinedo, F. y Lugo, L. (2012). Relación entre actividad física y obesidad en escolares. Revista cubana de Medicina general integral, 28(1), 34-41.
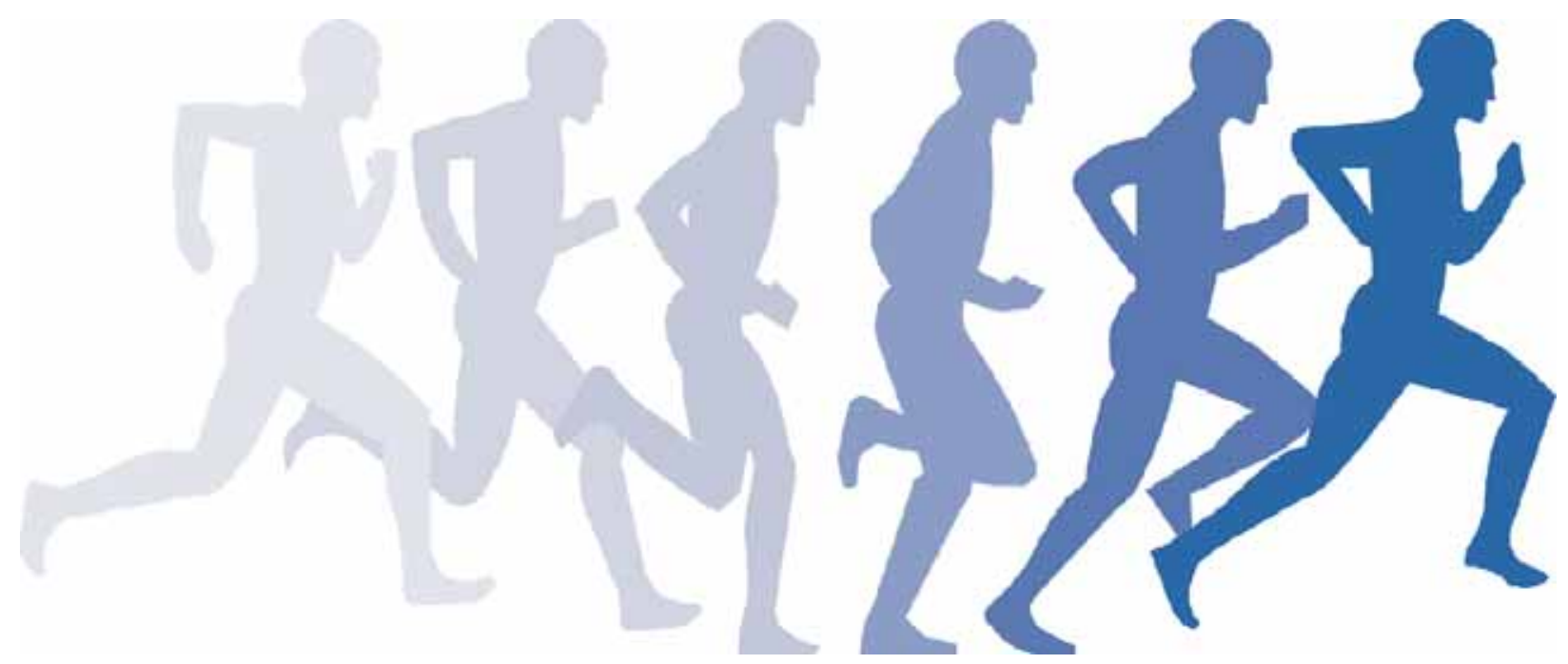